Dossiê "Saúde Única: Democratizando ciências, aprendizados e práticas"

Eixo temático: Saúde única e seus saberes

\title{
Desafios e propostas para a inclusão dos conceitos e práticas da "Saúde Única" no ambiente escolar
}

\section{Challenges and proposals for the inclusion of "One Health" concepts and} practices in the school environment

Gabriele Marisco ${ }^{12 *}$, Karine Brandão ${ }^{3}$, Sabrina Parente ${ }^{4}$, Taiara Alves ${ }^{5}$, Camila Parente ${ }^{1}$, Graça Regina Matias Ferreira ${ }^{6}$, Naura Angélica da Silva ${ }^{1}$, Margarida Pereira dos Santos ${ }^{1}$, Luiza da Silva Morais ${ }^{1}$

\footnotetext{
${ }^{1,2 *}$ Universidade Estadual do Sudoeste da Bahia - UESB, Vitória da Conquista, Bahia, Brasil 45026-145, gabrielemarisco@uesb.edu.br*, https:/orcid.org/0000-0002-8301-8673; amilacparente@gmail.com; naura_angelica@hotmail.com; bragamargarida13@gmail.com; easyriderluiza@gmail.com

${ }^{2 *}$ Programa de Pós-Graduação em Educação - UFBA, Faculdade de Educação, Bahia, Brasil 40110-100

3Centro Juvenil de Ciência e Cultura. karinebrandao.biologia@gmail.com

${ }^{4}$ Sartre Escola SEB, Lauro de Freitas, Bahia, Brasil, scparente@yahoo.com.br

${ }^{5}$ Secretaria Municipal de Vitória da Conquista, Bahia, Brasil, tai.sousa.bio@gmail.com

${ }^{6}$ Secretaria de Educação do Estado da Bahia, Salvador, Bahia, Brasil, gracamatiasf@gmail.com
}

\section{Resumo}

O presente artigo tem como objetivo, a partir de um relato de experiências, trabalhar a interdisciplinaridade na comunidade escolar por meio do prisma central da "Saúde Única", tendo em vista a importância dessa temática no aspecto social e a possibilidade de transpor diversas áreas do conhecimento por meio de experiências integradoras. Nesse sentido, através dos princípios norteadores baseados nas discussões que permearam, desde a educação em saúde e Saúde Única, até os desafios encontrados pela educação e a prática docente na busca da 
concretização de transformações mais efetivas na sociedade, práticas realizadas, como o Desafio e reflexões acerca da troca de conhecimentos, anseios e experiências que permeiam a formação docente inicial e continuada, de professores da educação básica e estudantes do curso de Licenciatura em Ciências Biológicas, foi factível o vislumbre de possibilidades concretas para a inserção dessa temática na comunidade escolar, de modo a reformular as práticas de ensino através de metodologias que tornem a aprendizagem mais significativa e atuante no contexto vivido.

Palavras chave: Saúde Única, Desafio Hackathon, educação em saúde, formação docente, interdisciplinaridade.

\begin{abstract}
This article aims, from an experience report, to work the interdisciplinarity in the school community through the central prism Unified Health, considering the importance of this theme in the social aspect and the possibility of transposing different areas of knowledge through of integrative experiences. In this sense, through the guiding principles based on the discussions that permeated, from health education and One Health, to the challenges faced by education and teaching practice in the search for the implementation of more effective transformations in society, practices carried out, such as the Challenge and reflections on the exchange of knowledge, anxieties and experiences that permeate the initial and continuing teacher education, of basic education teachers and students of the Licentiate Degree in Biological Sciences, it was possible to glimpse concrete possibilities for the insertion of this theme in the school community, in order to reformulate teaching practices through methodologies that make learning more meaningful and active in the lived context.
\end{abstract}

Keywords: One Health, Hackathon Challenge, health education, teacher training, interdisciplinarity.

\title{
1. Introdução
}

A interdisciplinaridade no ambiente escolar é uma proposta que visa um melhor entendimento das disciplinas correlacionando-as entre si, através da abordagem de conteúdos e temas contribuindo assim para uma aprendizagem estendida. Segundo Bonatto et al. (2012), a interdisciplinaridade na escola vem complementar as disciplinas gerando uma visão ampliada dos conceitos e fazendo com que os estudantes percebam que o mundo é diversificado em vários fatores formando assim uma complexidade. Ainda segundo os autores, para que aconteça a interdisciplinaridade não é necessário a exclusão de disciplinas, mas uma comunicação entre si 
e trazer para a atualidade as práticas que visa no processo de ensino e aprendizagem.

A "Saúde Única” é um exemplo de interdisciplinaridade, visto que reconhece a conexão entre saúde dos seres humanos, dos animais não-humanos e do meio ambiente, devendo ser trabalhadas em conjunto (Portal Saúde Única, 2021). Além disso, defende-se a utilização de estratégias e abordagens transdisciplinares e multissetoriais, sendo a escola um local apropriado para isso.

Considerando a dificuldade de abordar saúde única na escola, o objetivo desse trabalho foi apresentar um levantamento de propostas de professores da educação básica e estudantes do curso de Licenciatura em Ciências Biológicas, sobre caminhos que insiram a "Saúde Única" na escola, e a importância da interdisciplinaridade associada a atividades colaborativas.

\section{Material e Métodos}

Este trabalho apresenta-se como um relato de experiência, a respeito de um desafio realizado por 5 professores da educação básica e 5 discentes do curso de licenciatura em biologia, em agosto 2021, durante um curso de formação continuada. Para realizar o desafio, foram organizadas 3 equipes, sendo elas nomeadas "CriAtivas", "Profs Arteiros" e "Educa Saúde".

Inicialmente o desafio foi lançado: "Como estimular a prática da "Saúde Única” nas escolas?". Para isso, as equipes precisavam debater ideias, criar e desenvolver soluções, num prazo de 7 dias.

$\mathrm{Na}$ resolução do desafio, as equipes utilizaram os recursos digitais Google meet $^{\mathrm{TM}} \mathrm{e}$ Whatsapp $^{\mathrm{TM}}$ para levantamento de ideias e discussão entre os integrantes das respectivas equipes, formulando assim suas propostas, embasados na cientificidade e nas suas experiências quanto docentes e discentes, propostas essas que foram apresentadas na data acordada.

\section{Resultados}

Os dados apresentados neste relato são descritivos e reflexivos, e trazem à luz uma discussão sobre as dificuldades, desafios, sugestões e possíveis rotas para inclusão da Saúde 
única na escola. Além disso, apresenta uma proposta, que visa contribuir com a discussão dessa temática, com caminhos para inclusão desse tema na escola e consequentemente contribuir com a sociedade.

A partir do desafio "Como estimular a prática da saúde única nas escolas?” Foi possível observar que as resoluções das equipes se apresentaram de maneiras distinta, porém complementares. Para a Equipe dos Profs Arteiros, os principais desafios e entraves para alcançar este objetivo é o currículo, a gestão escolar, o estabelecimento de parcerias articulando diferentes áreas, como setores da saúde como vigilâncias e endemias, Programa Saúde na escola (PSE); setores do meio ambiente e da educação. Outro fator em destaque está relacionado com a formação continuada para professores e funcionários da escola, para execução de atividades interdisciplinares.

A equipe CriAtivas versaram sobre estratégias didáticas para abordar "Saúde Única" na escola, visando contemplar a individualidade de cada escola, perfil do anulado, condições socioeconômicas da comunidade escolar, a importância da interdisciplinaridade, e o papel fundamental da direção e coordenação escolar e a ruptura da resistência docente, para que essas práticas sejam mais efetivas.

Já a Equipe Educa Saúde, diante das circunstancias globais vivenciadas, trouxe a Saúde Única através do tema Zoonoses, como temática de fundamental importância para ser inserida na escola, vinculado à projetos interdisciplinares e participação da família e dos alunos de modo que, essa aprendizagem alcance toda a comunidade sendo assim, mais significativa na promoção de transformação social e saúde.

"Na intenção de elaborar, propor e divulgar ações que estimulam a prática da Saúde Única nas escolas do Brasil, um esquema norteador foi construído (Figura 1)”. 


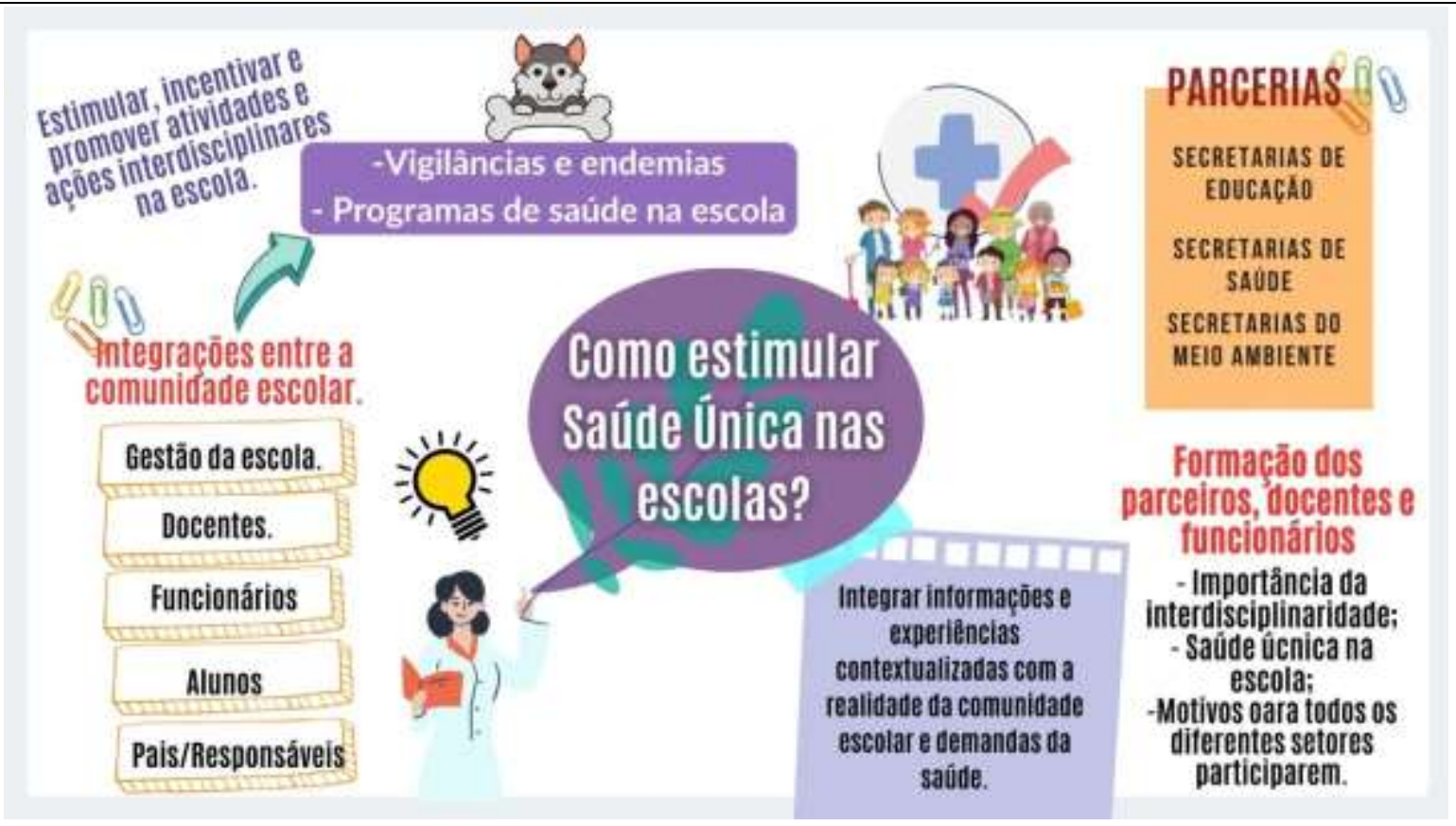

Figura 1. Proposta unificada de ações que estimulem a "Saúde Única” na escola.

A figura 2, exemplifica a participação de todos os setores envolvidos a partir da temática "Zoonoses: um problema de saúde única". Visto ser um problema que afeta muitas cidades do Brasil, e ser um tema que envolve o meio ambiente, saúde animal e a saúde humana.

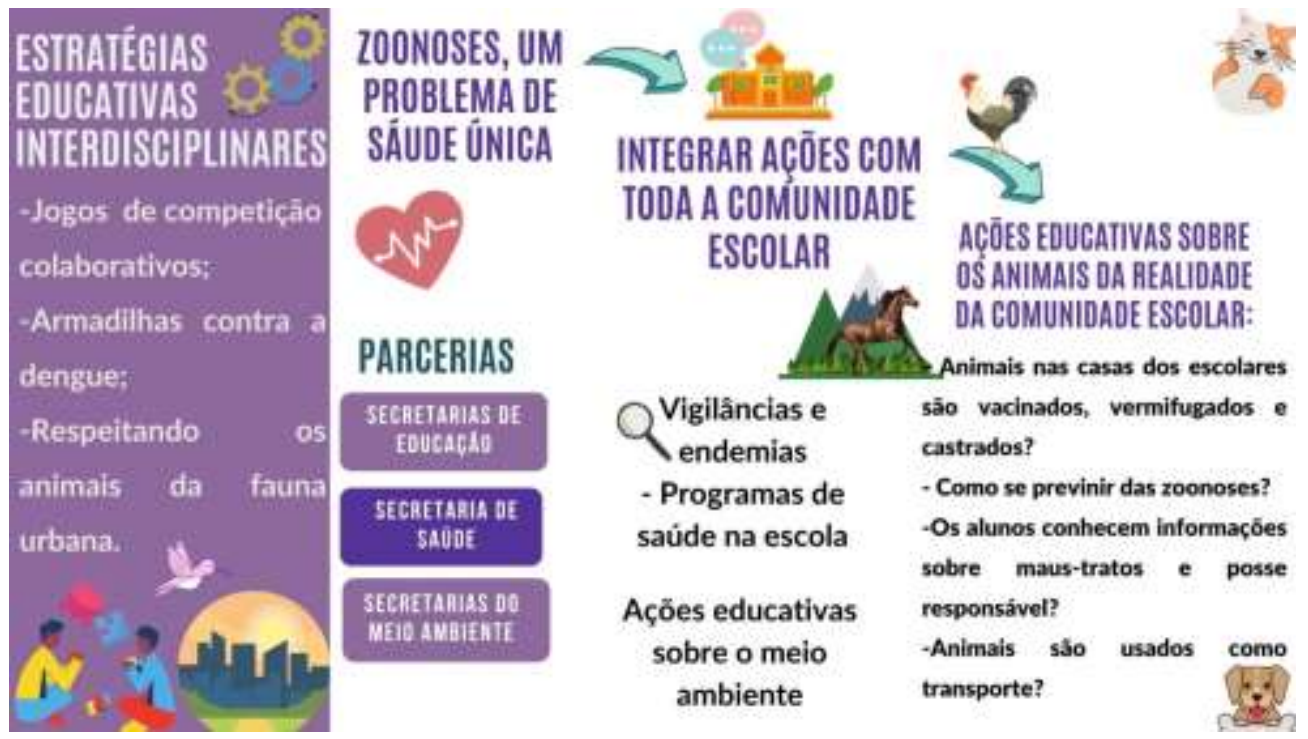

Figura 2. Exemplificação de uma ação interdisciplinar da temática "Zoonoses: um problema de saúde única". 


\section{Discussão}

Conforme previsto nos Parâmetros Curriculares Nacionais (PCN), a função da interdisciplinaridade na escola é incluir um ensino que faça com que os alunos sejam indagadores, críticos e argumentativos, saindo da passividade e aplicando esse conhecimento na prática. Para tanto, é necessário um educador interdisciplinar que traga renovação no ensino, na preparação das suas aulas, oportunizando aos alunos desenvolver senso crítico e participativo, visando a sua formação de uma maneira completa. Associado a isso, esse educador precisa buscar interação com outros professores a fim de fazer um elo entre as aulas, tornando-as mais ricas englobando diversos conhecimentos sem uma fragmentação do saber (UMBELINO \& ZABINI, 2014).

Segundo Sacristán (2000, p. 22) “o currículo faz parte, na realidade, de múltiplos tipos de práticas que não podem reduzir-se unicamente à prática pedagógica de ensino". Considerando que cada indivíduo é formado por um conjunto de saberes, o saber científico não pode descartar completamente o conhecimento popular, pois são esses conhecimentos que auxiliarão nas mediações didáticas do professor.

As metodologias ativas, utilizando tecnologias digitais, precisam estar inseridas na construção do currículo escolar, uma vez que ela consegue atingir cada indivíduo de uma maneira única, pois possibilita aulas dinâmicas e criativas favorecendo a construção de uma relação entre professor-aluno de maneira horizontal. O professor passa a ser um mediador do conhecimento, e começa a desempenhar um papel de motivador, o que favorece o aprendizado do educando.

Trazendo um viés para tratar da saúde única nas escolas na contemporaneidade, buscamos nesta seção dialogar sobre a possibilidade de trazer estratégias didáticas inovadoras, tais como o uso de ferramentas digitais, aulas práticas e atividades mão na massa, gamificação (jogos), desafios de forma a engajar e diversificar outras formas de trabalhar essa temática, de forma interdisciplinar, tão ampla em sala de aula com uso de metodologias ativas (MATTAR, 2013).

O uso dos recursos digitais, intensificados por conta da pandemia do Coronavírus, pode 
auxiliar na proposta de sensibilizar e disseminar o conhecimento da "Saúde Única" nas unidades escolares, trazendo novos desafios à educação. As redes sociais também podem ser utilizadas para disseminar a popularização do ensino (Santos \& Caputo, 2018) auxiliando inclusive na divulgação científica no contexto vivenciado.

Cabe aqui destacar que, ao utilizar essas estratégias didáticas, devemos observar a individualidade e as possibilidades pedagógicas e de infraestrutura de cada unidade escolar tendo como direção o perfil dos alunos e alunas, as condições socioeconômicas bem como as possibilidades de seus usos em sala de aula, de forma a favorecer o processo de aprendizagem sensível, pautado no sentir, pensar e agir mobilizando o corpo docente e a comunidade escolar para uma educação em "Saúde Única” lúdica e transformadora.

Outro aspecto importante para promoção da "Saúde Única" e execução de projetos na escola, é a necessidade do apoio integrado entre os setores da gestão pública do município, que parte do próprio conceito de "Saúde Única", o da articulação entre seres humanos (assistência social), animais e meio ambiente saudáveis, ou seja, a necessidade do estabelecimento de parcerias nos órgãos gestores desses elementos, como uma rede colaborativa de apoio à esses projetos, estimulando alianças entre os diferentes atores em diversos níveis da sociedade.

As estratégias-chave para promover a saúde, devem estar nas agendas das secretarias de meio ambiente, saúde, educação e assistência social, que são diretamente responsáveis pelo estabelecimento de políticas públicas e podem oferecer recursos para a criação de ambientes favoráveis à promoção da saúde, o fortalecimento de ações comunitárias, projetos realizados nas escolas, o desenvolvimento de habilidades pessoais e a reorientação dos próprios serviços de saúde (Pinto, 2020).

Um outro aspecto essencial para a implantação da "Saúde Única" nas escolas é a participação da família, é no ceio familiar onde se inicia a formação dos valores sociais e morais dos indivíduos, além de ser a porta de entrada para as primeiras aprendizagens de um indivíduo. As crianças começam sua formação em casa com o auxílio dos pais e cuidadores, mas ao chegar em idade escolar é perceptível um abandono desse acompanhamento dos pais para com seus filhos, o que acarreta grandes dificuldades para a escola no processo de educar. 
O acompanhamento familiar é essencial no aprender do aluno. Macedo (1994, p.199) afirma que com a participação da "família no processo de ensino aprendizagem, a criança ganha confiança vendo que todos se interessam por ela, e também porque você passa a conhecer quais são as dificuldades e quais os conhecimentos da criança”. Esclarecendo que todos alunos já possuem um conhecimento e que o conteúdo lecionado na escola deve ser trabalhado levando em consideração a experiência desse aluno.

O conhecimento do meio em que o aluno vive, é essencial para o seu desenvolvimento educacional, entendendo qual estrutura familiar ele vivencia e qual comunidade ele faz parte. Essas informações são importantes para percebermos esse aluno como indivíduo único e buscar nesses elementos, a participação da família nessa construção do conhecimento científico. Grinspun (2001, p.149) afirma que a prática do orientador, hoje deve colaborar em construir o conhecimento, facilitar as condições de aquisições desse conhecimento, promovendo as interações e toda a teia de relações que envolvem o sujeito e o meio. Dessa forma envolver a família nos processos educacionais, trazendo a importância da sua colaboração para a boa formação educacional e social dos seus filhos.

Associado às questões mencionadas acima, destacamos a formação continuada docente, visto que é um processo que proporciona desenvolvimento, exige continuidade e surge da necessidade dos professores buscarem novas práticas para a construção de conhecimentos por conta dos múltiplos e novos desafios do mundo contemporâneo. A formação continuada não se caracteriza como uma mera transmissão de técnicas prontas, mas à associação da prática docente e de suas peculiaridades relacionadas a um espaço, ao tempo e ao conhecimento, valorizando sempre os saberes do professor, embasados no trabalho cotidiano e no conhecimento do meio (Bezerra \& Oliveira, 2020).

É importante salientar, que o professor deve participar de inovações da escola de forma crítica, sendo essencial o fortalecimento do papel das lideranças o reforço dos trabalhos em grupos no interior da escola, de forma que promova a criação de vínculos e corresponsabilidades entre os docentes, que sustentem práticas e tentativas de mudanças com vistas a uma educação mais democrática. Para isso, no interior da escola não é necessário apenas 
à garantia nas condições de acesso, mas a criação de uma cultura escolar que estimule a autonomia consciente através de experiências integradoras que conectam todos os elementos do saber pedagógico e dão sentido aos mesmos, tornando a aprendizagem de fato significativa.

Assim, os efeitos da formação continuada na prática docente devem ser acompanhados e para isso é necessário que se observe as alterações na prática pedagógica a partir da proposta de inovação ofertada pelo programa de formação continuada, realização de analises das condições intra e extraescolares que beneficiam ou impossibilitam a efetivação, a incorporação e a manutenção de práticas pedagógicas sugeridas ao professor que passou pela formação (Trebien et al, 2020; Falsarella, 2004).

Diante das reflexões apontadas, foi factível o vislumbre de possibilidades concretas para a inserção dessa temática na comunidade escolar, de modo a reformular as práticas de ensino através de metodologias que tornem a aprendizagem mais significativa e atuante no contexto vivido.

\section{Referências}

Aranha, M. L. de A. (2002). Filosofia da Educação. 2. ed. São Paulo: Moderna, 302p.

Bezerra, C. M. da S.; \& Oliveira, E. dos S. (2020). Formação continuada do professor do ensino médio integrado: Concepções e importância. Revista Brasileira da Educação Profissional e Tecnológica, v. 1, n. 8, p. 1-15. https://doi.org/10.15628/rbept.2020.9281

Bonatto, A.; Barros, C. R.; Gemeli, R. A.; Lopes. et al. (2012). Interdisciplinaridade no ambiente escolar. IX ANPED SUL 2012: seminário de pesquisa em educação da região sul, http://www.ucs.br/etc/conferencias/index.php/anpedsul/9anpedsul/paper/viewFile/2414/501

Falsarella, A. M. (2004). Formação continuada e prática de sala de aula: os efeitos da formação continuada na atuação do professor. [Livro eletrônico]. $2^{\mathrm{a}}$ ed. Campinas, SP: Autores Associados. 236p.

Grinspun, M. P. S. (2006). A Orientação Educacional: conflito de paradigmas e alternativas para a escola. São Paulo; Cortez, 216p. http://bds.unb.br/handle/123456789/750

Sacristán, J. G. (2000). O currículo: uma reflexão sobre a prática. 3. ed. Porto Alegre: Penso, $352 p$. 
Macedo, R. M. (1994). A família diante das dificuldades escolares dos filhos. In: Bossa, N. A. \& Oliveira, V. B. Avaliação Psicopedagógica da Criança de Zero a Seis Anos. 2. ed. Petrópolis: Vozes, 226p.

Trebien, M. M.; Souza, W. R. de.; Oliveira, E. R. de.; Silva, J. L. da. (2020). Formação continuada de professores: uma epistemologia da prática. Ambiente: Gestão e Desenvolvimento, v. 13, n. 1, p. 91-102, https://doi.org/10.24979/359

Umbelino, M. \& Zabini, F. O. (2014). A importância da interdisciplinaridade na formação do docente. In: Seminário Internacional de Educação Superior, Sorocaba. Anais .... Sorocaba, Programa de Pós Graduação em Educação, 2014. p. 1-8.

\section{Direitos autorais (Copyrights)}

Financiamento: Esta pesquisa recebeu apoio da Proex/Geac/UESB.

Conflitos de interesse: Não houve qualquer conflito de interesse por parte das autoras.

Aprovação do comitê de ética: $O$ trabalho foi aprovado pelo Comitê de Ética em Pesquisa, envolvendo seres humanos no Brasil, CAAE: 33615220.1.0000.0055.

Disponibilidade dos dados de pesquisa: Todos os dados referentes a esta pesquisa estão disponibilizados no manuscrito.

Contribuição dos autores (todas as autoras participaram de todo o processo de construção do artigo). 